\title{
Evaluation of prognostic factors in patients with therapy-related acute myeloid leukemia
}

\author{
Sang Hyuk Park, Hyun-Sook Chi, Young-Uk Cho, Seongsoo Jang, Chan-Jeoung Park \\ Department of Laboratory Medicine, University of Ulsan College of Medicine, Asan Medical Center, Seoul, Korea
}

p-ISSN 2287-979X / e-ISSN 2288-0011 http://dx.doi.org/10.5045/br.2013.48.3.185 Blood Res 2013;48:185-92.

Received on May 17, 2013

Revised on July 1, 2013

Accepted on July 12, 2013

\author{
Correspondence to \\ Hyun-Sook Chi, M.D., Ph.D. \\ Department of Laboratory Medicine, \\ University of Ulsan College of Medicine, \\ Asan Medical Center, 88, Olympic-ro \\ 43-gil, Songpa-gu, Seoul 138-736, Korea \\ Tel: + 82-2-3010-4502 \\ Fax: +82-2-478-0884 \\ E-mail: hschi@amc.seoul.kr \\ (C) 2013 Korean Society of Hematology
}

\section{Background}

Therapy-related AML (t-AML) occurs as a late complication of chemotherapy administered to treat a prior disorder. Prognostic factors affecting the clinical outcome in t-AML have not yet been clearly defined; therefore, we evaluated these factors in this study.

\begin{abstract}
Methods
Forty-eight patients diagnosed with t-AML within the past 10 years were enrolled, and their chemotherapy regimens categorized into 4 groups: alkylating agents (AK) only, topoisomerase II inhibitors (TI) and AK, TI only, and others. The prognostic factors affecting clinical outcome were evaluated.
\end{abstract}

\begin{abstract}
Results
Five (10.4\%), 21 (43.8\%), 9 (18.8\%), and 13 (27.0\%) patients were treated with AK only, AK and TI, TI only, and others, respectively. Patients with an AML M3 phenotype showed significantly longer overall survival (OS; 55.1 vs. 14.3 months, $P=0.040$ ) and disease-free survival (DFS; 61.2 vs. 17.5 months, $P=0.049$ ) than other phenotypes. In contrast, patients with a complex karyotype showed significantly shorter OS (7.9 vs. 31.3 months, $P=0.008)$ and DFS (9.5 vs. 38.6 months, $P=0.046)$; additionally, patients with chromosome 5 or 7 abnormalities showed significantly shorter OS (9.1 vs. 30.7 months, $P=0.011$ ) than other phenotypes. Only the presence of a complex karyotype or AML M3 phenotype retained prognostic impact in a multivariate analysis.
\end{abstract}

Conclusion

Only the AML M3 phenotype was identified as having a good prognosis, and this might suggest that it exhibits unique clinical features in t-AML patients. Moreover, our findings indicated that karyotype was the strongest prognostic indicator and predicted a poor prognosis for t-AML patients with a complex karyotype.

Key Words Prognosis, Therapy, Related, AML

\section{INTRODUCTION}

The clinical manifestation of therapy-related AML ( $\mathrm{t}-$ $\mathrm{AML}$ ) can generally be divided into 2 groups: those who receive alkylating agents $(\mathrm{AK})$ and those who receive topoisomerase II inhibitors (TI). Patients with t-AML related to AK are characterized by a long interval between therapy and AML development and are associated with cytogenetic abnormalities involving chromosome 5 and 7. Patients with $\mathrm{t}$-AML related to TI present with a shorter latency and are associated with karyotype aberrations involving chromo- some 11 and 21 [1-5].

Overall, the prognosis of t-AML is worse than de novo AML, with a 5-year survival rate of less than $10 \%[6,7]$. In contrast to de novo AML, in which various prognostic factors have been clearly defined [8,9], a comprehensive study focused on the identification of independent prognostic factors in t-AML has been lacking. Although several studies have reported that karyotype is an independent prognostic parameter in t-AML, these studies focused only on cytogenetic abnormalities and did not include molecular aberrations, cytotoxic therapy regimens, or hemograms as prognostic indicators [10-12]. Additionally, a recently published 
paper reviewed clinical and cytogenetic characteristics of 39 patients diagnosed with therapy-related myeloid neoplasms from a single center, but this study did not focus on t-AML nor the prognostic factors [13]. In this study, we performed a comprehensive evaluation to identify the independent prognostic factors in t-AML.

\section{MATERIALS AND METHODS}

\section{Patient selection and treatment}

A total of $48 \mathrm{t}$-AML patients were identified in a retrospective, systematic review of electronic medical records of patients diagnosed with AML at Asan Medical Center, Seoul, Korea from January 2001 to December 2011. All patients received induction chemotherapy consisting of cytarabine and daunorubicin. The therapeutic regimen included continuous intravenous infusion of $200 \mathrm{mg} / \mathrm{mL}$ per day $(100$ $\mathrm{mg} / \mathrm{mL}$ per day for patients aged $>60$ years of age) of cytarabine from day 1 to day 7 , and $45 \mathrm{mg} / \mathrm{mL}$ per day of daunorubicin from day 1 to day 3. Complete remission (CR) was defined as the presence of less than 5\% blasts with $>20 \%$ cellularity in a bone marrow (BM) aspirate after induction chemotherapy. Relapse was defined as the presence of more than $5 \%$ leukemic blasts in bone marrow aspirates in patients with a previous CR state. Relapsed patients received the same induction chemotherapy regimen as that used at initial diagnosis. Depending on the patient's age and the availability of a suitable donor, patients received allogeneic stem cell transplantation (SCT) as close to the time of CR status confirmation as possible.

Patient classification and comparison of clinical presentation

The 48 t-AML patients were categorized into 4 groups according to chemotherapeutic regimen: AK only (5 patients), AK and TI (21 patients), TI only ( 9 patients), and others (13 patients). Clinical data for gender, age, FrenchAmerican-British (FAB) phenotype, hemogram results, FLT3 ITD mutation status, karyotype, CR rate, relapse rate, death rate, and date of allogeneic SCT were compared among the 4 patient groups. Patients who were classified as having chromosome $5 / 7$ or $11 / 21$ abnormalities were included in the chromosome $5 / 7$ or $11 / 21$ abnormality group, regardless of whether the patient had a complex karyotype or not. Similarly, patients were categorized into 2 groups with respect to the presence of a complex karyotype, irrespective of which, if any, chromosomal abnormality was present.

From these data, we calculated the prognostic markers, overall survival (OS), and disease-free survival (DFS). OS was defined as the time from diagnosis to death or, alternatively, last follow-up. DFS was defined as the time from CR to relapse for patients who experienced a relapse, death for non-relapsed patients who did not survive, or the time of the last follow-up for surviving patients who did not experience a relapse. If the patients received allogeneic SCT, the initial CR date for the calculation of DFS was replaced by the SCT date.

\section{Comparison of prognosis}

The impact of clinical variables on OS and DFS was evaluated separately. Variables included in the analysis were chemotherapy regimens, BM blast percentage, FLT3 ITD mutation status, presence of chromosome $5 / 7$ or $11 / 21$ abnormalities, presence of a complex karyotype, presence of a FAB M3 phenotype, and performance status of allogeneic SCT. The BM blast cell percentage comparison of prognosis was analyzed using the median value of BM blasts (61.1\%) as a cutoff for the differentiation of 2 groups. Multivariate analysis was performed to confirm variables that had a significant independent prognostic impact during univariate analysis. Age, BM blasts, performance status of allogeneic SCT, FLT3 ITD mutation status, presence of chromosome 5/7 abnormalities, presence of a complex karyotype, and FAB M3 phenotype were included as covariables in the multivariate analysis.

\section{Statistical analysis}

Pearson chi-square or Fisher's exact tests were used to compare categorical variables with respect to the 4 chemotherapy groups. The Kruskal-Wallis test was used to compare continuous variables with respect to the 4 chemotherapy groups. Estimates of survival (OS and DFS) were made using the Kaplan-Meier method and were compared using a log-rank test. Multivariate analyses of OS and DFS were performed using Cox's proportional hazards model. For all analyses, tests were two-tailed and $P$ values $\leq 0.05$ were considered statistically significant. All calculations were performed using SPSS 13.0.1 for Windows (SPSS Inc., Chicago, IL, USA).

\section{RESULTS}

\section{Comparison of clinical presentation}

Patients developed t-AML with a median latency of 36.3

Table 1. Distribution of primary neoplastic disorders in enrolled patients.

\begin{tabular}{lc}
\hline \multicolumn{1}{c}{ Disorder } & $\mathrm{N}(\%)$ \\
\hline Breast cancer & $11(22.9 \%)$ \\
Non-Hodgkin's lymphoma & $7(14.6 \%)$ \\
Colorectal cancer & $4(8.3 \%)$ \\
Hepatocellular carcinoma & $4(8.3 \%)$ \\
Cervical cancer & $3(6.3 \%)$ \\
Endometrial cancer & $3(6.3 \%)$ \\
Stomach cancer & $3(6.3 \%)$ \\
Bladder cancer & $2(4.1 \%)$ \\
Other ${ }^{a)}$ & $11(22.9 \%)$ \\
Total & $48(100.0 \%)$ \\
\hline
\end{tabular}

${ }^{a}$ Other disorders include (1 patient each): esophageal cancer, cholangiocarcinoma, non-small cell lung cancer, malignant germinoma, medulloblastoma, ovarian cancer, prostate cancer, thyroid cancer, Ewing's sarcoma, high grade ependymoma, and renal cell carcinoma. 
months. Breast cancer (22.9\%) was the most common primary neoplastic disorder, followed by non-Hodgkin's lymphoma (14.6\%), colorectal cancer, and hepatocellular carcinoma $(8.3 \%$ each) (Table 1$)$. The distribution of gender, age, FAB phenotype, hemogram results, BM blast percentage, allogeneic SCT performance rates, and FLT3 ITD mutation positivity at diagnosis of t-AML was not significantly different among the 4 patient groups. Additionally, there were no significant differences in the frequency of chromosome 5 or 7 abnormalities, chromosome 11 or 21 , and presence

Table 2. Comparison of clinical presentation with respect to chemotherapeutic regimen.

\begin{tabular}{|c|c|c|c|c|c|}
\hline \multirow[b]{2}{*}{ Variable } & \multicolumn{4}{|c|}{ Chemotherapeutic regimen } & \multirow[b]{2}{*}{$P$} \\
\hline & $\begin{array}{c}\text { AK only } \\
(\mathrm{N}=5)\end{array}$ & $\begin{array}{l}\mathrm{AK}+\mathrm{TI} \\
(\mathrm{N}=21)\end{array}$ & $\begin{array}{l}\text { TI only } \\
(\mathrm{N}=9)\end{array}$ & $\begin{array}{c}\text { Others } \\
(\mathrm{N}=13)\end{array}$ & \\
\hline Gender (M:F) & $4: 1$ & $5: 16$ & $6: 3$ & $6: 7$ & $0.053^{\mathrm{a})}$ \\
\hline Median age (years) (range) & $37.0(17.0-64.0)$ & $57.0(39.0-73.0)$ & $62.0(6.0-80.0)$ & $62.0(47.0-81.0)$ & $0.075^{\mathrm{b})}$ \\
\hline \multicolumn{6}{|l|}{ FAB classification $(\%)$} \\
\hline M1 & 0.0 & 9.6 & 0.0 & 7.7 & \multirow{7}{*}{$0.399^{\mathrm{a})}$} \\
\hline M2 & 0.0 & 19.0 & 0.0 & 15.4 & \\
\hline M3 & 20.0 & 38.1 & 22.2 & 38.4 & \\
\hline M4 & 20.0 & 14.3 & 33.4 & 7.7 & \\
\hline M5 & 60.0 & 19.0 & 11.1 & 15.4 & \\
\hline M6 & 0.0 & 0.0 & 22.2 & 7.7 & \\
\hline M7 & 0.0 & 0.0 & 11.1 & 7.7 & \\
\hline Median WBC $\left(\times 10^{9} / \mathrm{L}\right)$ (range) & $9.7(2.6-84.2)$ & $5.9(0.8-133.0)$ & $2.4(0.9-22.9)$ & $3.1(1.2-67.6)$ & $0.339^{b)}$ \\
\hline Median $\mathrm{Hb}(\mathrm{g} / \mathrm{dL})$ (range) & $8.6(7.5-10.2)$ & $8.9(7.3-11.8)$ & $8.6(7.6-10.4)$ & $9.7(6.9-11.6)$ & $0.755^{\mathrm{b})}$ \\
\hline Median PLT $\left(\times 10^{9} / \mathrm{L}\right)($ range $)$ & $49.0(15.0-90.0)$ & $46.0(20.0-170.0)$ & $41.0(11.0-356.0)$ & $77.0(6.0-358.0)$ & $0.174^{\mathrm{b})}$ \\
\hline Median interval to AML (months) (range) & $64.8(50.4-111.3)$ & $29.7(5.9-135.7)$ & $16.7(3.9-78.8)$ & $32.6(1.7-154.3)$ & $0.041^{b)}$ \\
\hline Median BM blasts (\%) (range) & $58.0(32.2-77.6)$ & $49.8(21.2-96.0)$ & $60.4(28.8-91.8)$ & $62.8(21.4-92.4)$ & $0.927^{b)}$ \\
\hline FLT3 ITD mutation rates (\%) & 0.0 & 23.8 & 11.1 & 7.7 & $0.455^{\mathrm{a})}$ \\
\hline \multicolumn{6}{|l|}{ Cytogenetic abnormality (\%) } \\
\hline Ch5 or Ch7 & 60.0 & 19.0 & 33.3 & 38.5 & $0.292^{\mathrm{a})}$ \\
\hline Ch11 or Ch21 & 20.0 & 33.3 & 22.2 & 30.8 & $0.910^{\mathrm{a})}$ \\
\hline Complex karyotype (\%) & 60.0 & 19.0 & 33.3 & 30.8 & $0.358^{\mathrm{a})}$ \\
\hline CR rate $(\%)$ & 60.0 & 66.7 & 44.4 & 61.5 & $0.778^{\text {a) }}$ \\
\hline Relapse rate (\%) & 33.3 & 14.3 & 40.0 & 37.5 & $0.257^{\mathrm{a})}$ \\
\hline Allogeneic SCT performance rate (\%) & 40.0 & 23.9 & 11.1 & 46.2 & $0.295^{\mathrm{a})}$ \\
\hline
\end{tabular}

$P$ value was calculated from the ${ }^{\text {a) }} \mathrm{Chi}$-square test and ${ }^{\text {b) }}$ Kruskal-Wallis test.

Abbreviations: AK, alkylating agent; TI, topoisomerase II inhibitor; FAB, French-American-British; PLT, platelet; BM, bone marrow; FLT3 ITD, fms-related tyrosine kinase 3 gene internal tandem duplications; Ch, chromosome; CR, complete remission; SCT, stem cell transplantation.
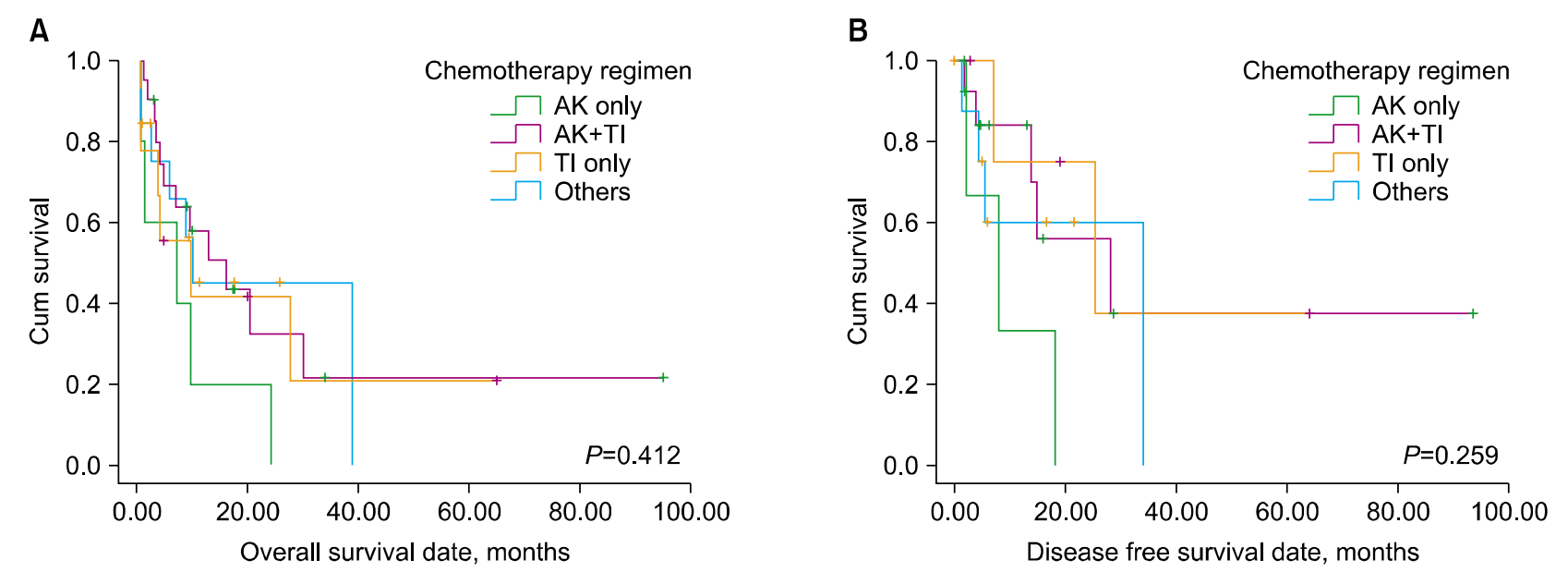

Fig. 1. The overall survival (A) and disease-free survival (B) of patients with therapy-related AML. Patients were categorized into 4 chemotherapeutic regimen groups for the treatment a primary neoplastic disorder: alkylating agents (AK) only, topoisomerase II inhibitors (TI) and AK, TI only, and others. 
of a complex karyotype among the 4 patient groups. Similarly, $\mathrm{CR}$ and relapse rates were not significantly different. The AK only group showed a significantly longer latency (median, 64.8 months) and the TI only group showed a significantly shorter latency (median, 16.7 months) compared to the other groups ( $P=0.041$; Table 2$)$.

\section{Comparison of prognosis}

The 4 chemotherapy regimens did not significantly influence the clinical outcome (Fig. 1). Additionally, prognosis was unaffected by BM blast percentage, FLT3 ITD mutation status, allogeneic SCT performance, and the presence of chromosome 11 or 21 abnormalities at the time of t-AML diagnosis. However, patients with chromosome 5 or 7 abnormalities showed a significantly shorter OS (9.1 vs. 30.7 months, $P=0.011)$ than those without aberrations, even though differences in DFS were not statistically significant $(P=0.498$; Fig.
2). Notably, patients with a complex karyotype at the time of t-AML diagnosis showed a significantly shorter OS (7.9 vs. 31.3 months, $P=0.008)$ and DFS (9.5 vs. 38.6 months, $P=0.046$ ) than those without a complex karyotype (Fig. 3). In addition, patients with an FAB M3 phenotype showed a significantly longer $O S$ (55.1 vs. 14.3 months, $P=0.040$ ) and DFS (61.2 vs. 17.5 months, $P=0.049)$ than those with other FAB phenotypes (Fig. 4 and Table 3).

\section{Multivariate analysis}

The presence of an FAB M3 phenotype possessed an independently good prognostic impact on both OS (hazard ratio [HR] 0.121, $P=0.012$ ) and DFS (HR 0.151, $P=0.038$ ) when age, BM blast percentage, performance status of allogeneic SCT, FLT3 ITD mutation status, presence of chromosome 5/7 abnormalities, and presence of a complex karyotype were adjusted. In contrast, the presence of a complex karyotype
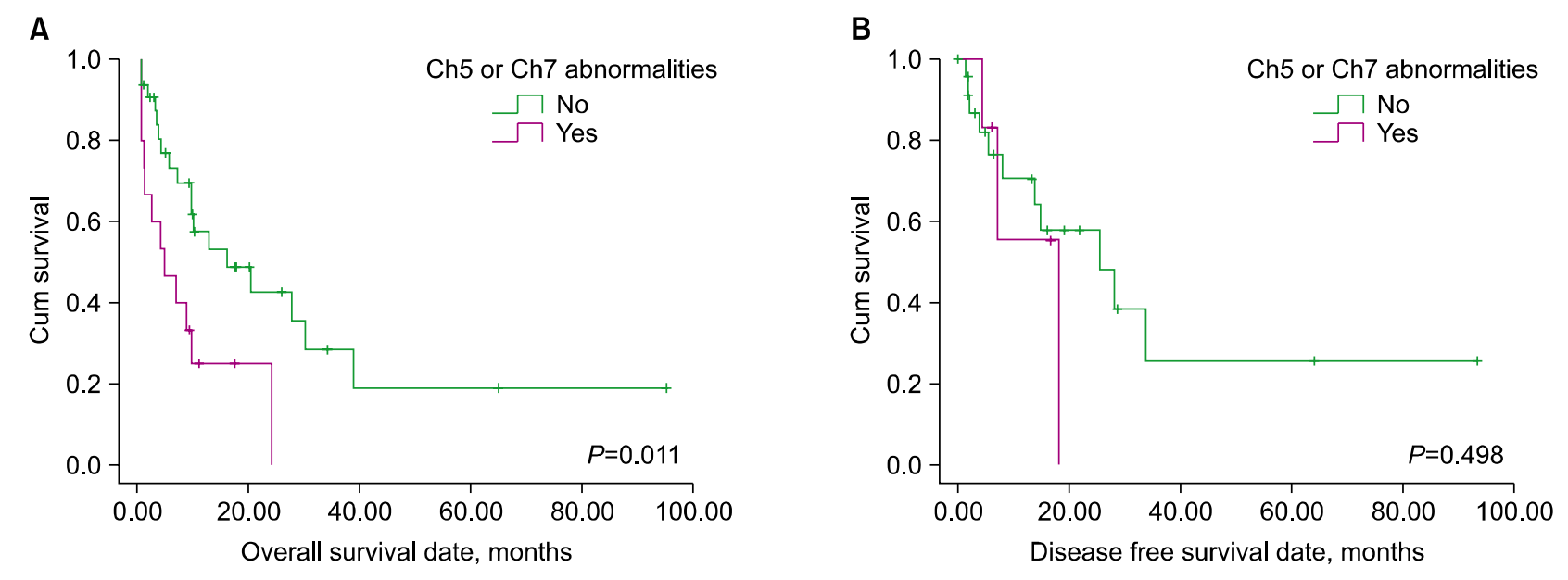

Fig. 2. The overall survival (A) and disease-free survival (B) of patients with therapy-related AML according to the presence of chromosome 5 or 7 abnormalities at diagnosis.
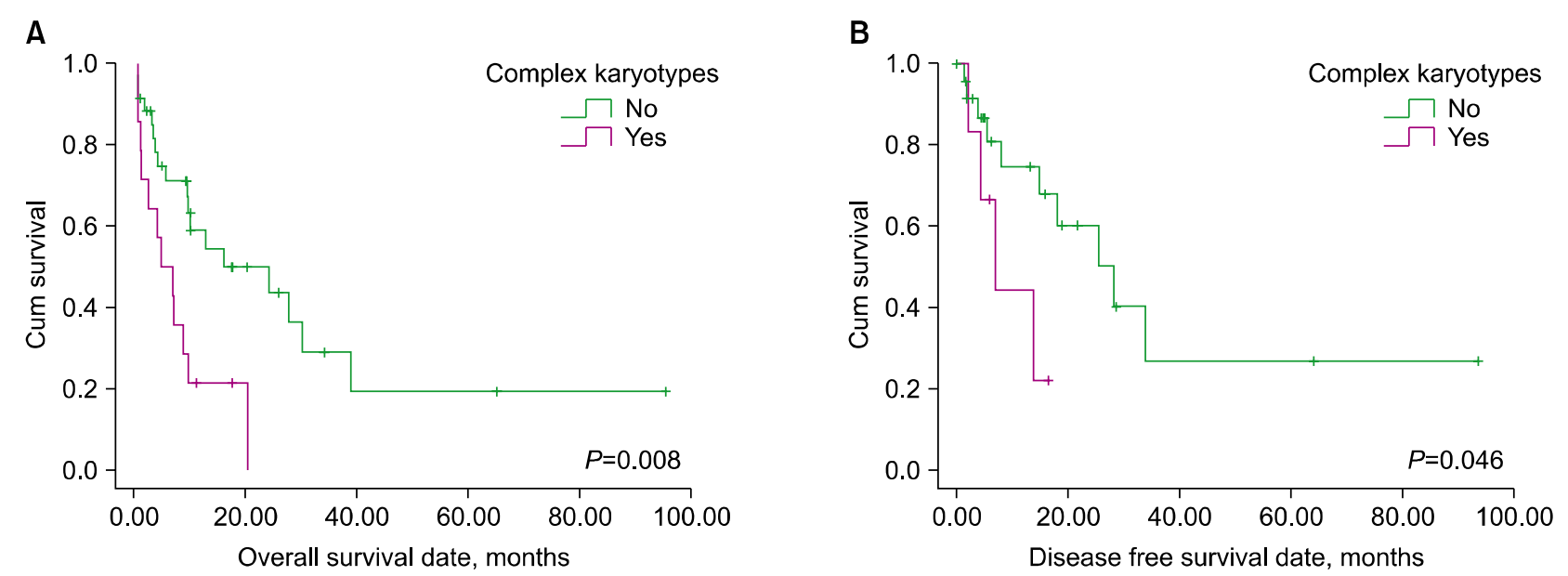

Fig. 3. The overall survival (A) and disease-free survival (B) of patients with therapy-related AML according to the presence of a complex karyotype at diagnosis. 

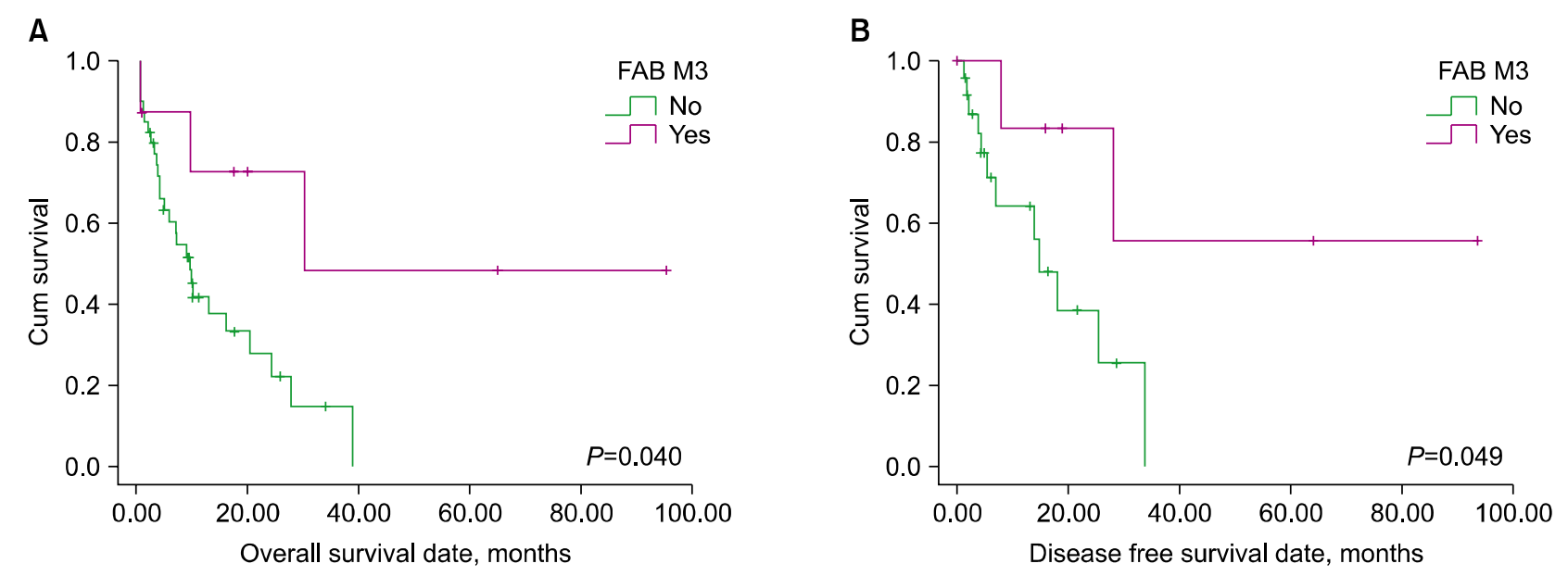

Fig. 4. The overall survival (A) and disease-free survival (B) of patients with therapy-related AML according to the presence of the FAB M3 phenotype at diagnosis.

Table 3. Comparison of overall and disease free survival.

\begin{tabular}{|c|c|c|c|c|}
\hline Variable & $\begin{array}{l}\text { Mean overall survival } \\
\text { (months) }(95 \% \mathrm{Cl})\end{array}$ & $P^{\mathrm{b})}$ & $\begin{array}{l}\text { Mean disease free survival } \\
\text { (months) }(95 \% \mathrm{Cl})\end{array}$ & $P^{\mathrm{b})}$ \\
\hline \multicolumn{5}{|l|}{ Chemotherapy regimen } \\
\hline AK only & $8.8(0.5-17.1)$ & \multirow{4}{*}{0.412} & $9.4(0.3-18.5)$ & \multirow{4}{*}{0.259} \\
\hline $\mathrm{AK}+\mathrm{TI}$ & $30.3(10.7-50.0)$ & & $44.7(16.1-73.2)$ & \\
\hline TI only & $21.8(4.0-39.6)$ & & $35.4(9.7-61.0)$ & \\
\hline Others & $20.5(12.8-36.5)$ & & $21.9(9.5-34.2)$ & \\
\hline \multicolumn{5}{|l|}{ BM blasts ${ }^{\mathrm{a})}$} \\
\hline$\leq 61.1 \%$ & $15.2(10.0-20.3)$ & \multirow[t]{2}{*}{0.868} & $15.1(9.4-20.8)$ & \multirow[t]{2}{*}{0.085} \\
\hline$>61.1 \%$ & $29.1(11.8-46.3)$ & & $46.3(19.9-72.7)$ & \\
\hline \multicolumn{5}{|l|}{ FLT3 ITD mutation } \\
\hline Absence & $18.5(10.3-26.8)$ & \multirow[t]{2}{*}{0.510} & $24.9(12.1-37.7)$ & \multirow[t]{2}{*}{0.630} \\
\hline Presence & $21.7(11.7-31.7)$ & & $26.8(24.2-29.5)$ & \\
\hline \multicolumn{5}{|c|}{ Ch11 or Ch21 abnormality } \\
\hline Absence & $25.2(12.3-38.2)$ & \multirow[t]{2}{*}{0.454} & $33.0(14.9-51.1)$ & \multirow[t]{2}{*}{0.667} \\
\hline Presence & $13.6(6.1-21.1)$ & & $17.8(8.3-27.3)$ & \\
\hline \multicolumn{5}{|l|}{ Ch5 or Ch7 abnormality } \\
\hline Absence & $30.7(15.3-46.2)$ & \multirow[t]{2}{*}{0.011} & $36.7(16.9-56.4)$ & \multirow[t]{2}{*}{0.498} \\
\hline Presence & $9.1(4.2-14.1)$ & & $12.8(5.7-19.8)$ & \\
\hline \multicolumn{5}{|l|}{ Complex karyotype } \\
\hline Absence & $31.3(15.7-46.9)$ & \multirow[t]{2}{*}{0.008} & $38.6(18.2-58.9)$ & \multirow[t]{2}{*}{0.046} \\
\hline Presence & $7.9(4.1-11.9)$ & & $9.5\left(4.8^{-14.1)}\right.$ & \\
\hline \multicolumn{5}{|l|}{ FAB M3 } \\
\hline Absence & $14.3(9.6-21.7)$ & \multirow[t]{2}{*}{0.040} & $17.5(11.2-23.8)$ & \multirow[t]{2}{*}{0.049} \\
\hline Presence & $55.1(21.8-88.6)$ & & $61.2(26.4-95.9)$ & \\
\hline \multicolumn{5}{|l|}{ Allogeneic SCT } \\
\hline Absence & $21.1(7.5-34.7)$ & \multirow{2}{*}{0.145} & $41.2(15.9-66.4)$ & \multirow{2}{*}{0.642} \\
\hline Presence & $25.5(17.4-33.6)$ & & $21.2(13.3-29.1)$ & \\
\hline
\end{tabular}

had an independently adverse prognostic impact on both OS (HR 2.877, $P=0.041$ ) and DFS (HR 3.569, $P=0.047$ ) when the same clinical variables were adjusted. The performance of allogeneic SCT had an independently good prognostic impact on OS (HR $0.145, P=0.018$ ), but not on DFS (HR $0.651, P=0.729)$. Interestingly, the presence of chromosome 
Table 4. Multivariate analysis of overall and disease free survival.

\begin{tabular}{|c|c|c|c|c|c|c|}
\hline \multirow{2}{*}{ Clinical variables } & \multicolumn{3}{|c|}{ Overall survival } & \multicolumn{3}{|c|}{ Disease free survival } \\
\hline & $\mathrm{HR}(95 \% \mathrm{Cl})$ & $P$ & Prognostic impact & $\mathrm{HR}(95 \% \mathrm{Cl})$ & $P$ & Prognostic impact \\
\hline Age & $1.019(0.984-1.055)$ & 0.288 & NS & $1.085(0.939-1.255)$ & 0.269 & NS \\
\hline BM blasts & $0.998(0.981-1.016)$ & 0.859 & NS & $0.999(0.965-1.033)$ & 0.950 & NS \\
\hline Allogeneic $\mathrm{SCT}^{\mathrm{a})}$ & $0.145(0.037-0.574)$ & 0.018 & Good if present & $0.651(0.058-7.342)$ & 0.729 & NS \\
\hline FLT3 ITD mutation ${ }^{\text {a) }}$ & $1.438(0.428-4.835)$ & 0.557 & NS & $2.665(0.305-23.303)$ & 0.376 & NS \\
\hline$F A B M 3^{a)}$ & $0.121(0.024-0.625)$ & 0.012 & Good if present & $0.151(0.011-0.878)$ & 0.038 & Good if present \\
\hline Ch5 or ch7 abnormality ${ }^{a)}$ & $1.435(0.427-4.829)$ & 0.559 & NS & $1.239(0.727-2.024)$ & 0.651 & NS \\
\hline Complex karyotype $^{\text {a) }}$ & $2.877(1.345-5.269)$ & 0.041 & Poor if present & $3.569(1.212-14.168)$ & 0.047 & Poor if present \\
\hline
\end{tabular}

${ }^{\text {a) }}$ The hazard ratio for the allogeneic SCT, FLT3 ITD mutation, FAB M3, chromosome 5 or chromosome 7 abnormality, and complex karyotype represents relative risk for overall and disease free survival in cases with presence of these variables compared to absence.

Abbreviations: $\mathrm{HR}$, hazard ratio; Cl, confidence interval; BM, bone marrow; SCT, stem cell transplantation; FLT3 ITD, fms-related tyrosine kinase 3 gene internal tandem duplications; FAB, French-American-British; Ch, chromosome; NS, not significant.

5 or 7 abnormalities did not function as a negative prognostic factor on either OS (HR 1.435, $P=0.559$ ) or DFS (HR 1.239, $P=0.651)$ in multivariate analysis. Other variables such as age, BM blasts, and FLT3 ITD mutation status also did not possess any prognostic impact on either OS or DFS (Table $4)$.

\section{DISCUSSION}

In de novo AML development, specific cytogenetic abnormalities and molecular aberrations such as FLT3 ITD, NPM1, and $C E B P A$ mutations have been firmly identified as prognostic indicators. In addition, $D N M T 3 A, I D H 1$, and $I D H 2$ mutations are currently believed to have a potential prognostic significance $[8,9]$. In contrast, studies focused on the identification of prognostic markers in t-AML have been limited and, except for karyotype, very little information is available [10-12]. In our study, we aimed to identify independent prognostic factors in t-AML while taking into consideration various clinical parameters as well as molecular and cytogenetic aberrations.

In our study, the median latency from the time of diagnosis of a primary neoplastic disorder to the development of $\mathrm{t}-\mathrm{AML}$ was 36.3 months, which is shorter than that reported in previous studies (median, 47-50 months) [12, 13]. Moreover, our data showed that the median latency of t-AML as 64.8 and 16.7 months in patients receiving AK only and TI only, respectively. Given that the use of $\mathrm{AK}$ and TI correlates with chromosomal 5 or 7 and 11 or 21 abnormalities, our results support the previous concept that the latency of $\mathrm{t}$-AML differs with respect to the chemotherapy regimen used [14]. In our patient cohort, chromosome 5 or 7 abnormalities were detected in 15/48 (31.3\%) t-AML patients; among them, 14 (93.3\%) showed either a whole or partial deletion of chromosome 5 or 7 . Chromosome 11 or 21 abnormalities were present in $14 / 48$ (29.2\%) of t-AML patients, and $10(71.4 \%)$ of these patients showed a balanced translocation involving 11q23. These results also correlate with previous studies [13, 14].

Previous studies that analyzed prognostic factors in $\mathrm{t}-\mathrm{AML}$ concluded that karyotype was the most important prognostic parameter, and both favorable and unfavorable karyotypes have been identified, which have the same prognostic impact for $\mathrm{t}-\mathrm{AML}$ as that for their de novo counterparts [11-14]. Our study demonstrated that there are no prognostic differences with respect to chemotherapy regimens in patients with t-AML. BM blast cell percentages and FLT3 ITD mutation status, which were known to have significant prognostic impact in patients with de novo AML, also did not possess any prognostic impact in $\mathrm{t}-\mathrm{AML}$ patients. Despite the apparently poor prognostic impact of increasing age and favorable performance of allogeneic SCT in de novo AML, the present study indicated that age did not have any prognostic impact on either OS or DFS. Additionally, the performance of allogeneic SCT was only found to be a favorable prognostic indicator for OS. Although the presence of chromosome 5 or 7 abnormalities, a complex karyotype, and FAB M3 phenotype had significant prognostic value in the univariate analysis (the former two are poor and the latter one is a good prognostic indicator), which is similar to de novo AML, multivariate analysis did not indicate that chromosome 5 or 7 abnormalities correlated with a poor prognosis. These results suggest that $\mathrm{t}-\mathrm{AML}$ might have unique prognostic features compared to de novo AML, and the establishment of a specific prognostic model for t-AML may be required.

Although data from an international study on prognostic factors in $\mathrm{t}$-AML is available, an analysis focused on $\mathrm{t}$-AML in the Korean population has been limited to 2 studies that reviewed demographic and clinical findings in $\mathrm{t}$-AML/MDS (myelodysplastic syndrome) patients $[13,15]$. A study that focused on 39 patients with $\mathrm{t}-\mathrm{AML} / \mathrm{MDS}$ reported that breast cancer was the most common primary solid tumor (23.1\%). The same study also demonstrated a shorter latency interval in patients with balanced rearrangements than in patients with the loss of chromosome 5 or 7 [13]. These findings are consistent with the results of the present study; however, the previous study reported that balanced translocations were 
frequently detected in patients who had undergone therapy to treat a solid tumor and that most patients with balanced translocations developed $\mathrm{t}-\mathrm{AML}$ rather than $\mathrm{t}-\mathrm{MDS}$ [13]. This trend could not be confirmed in the present study, since our study population did not include t-MDS patients. Another study focused on 12 patients who developed therapy-related acute leukemia after treatment for breast cancer and reported that $67 \%$ of patients had balanced translocations involving $11 \mathrm{q} 23$ and that patients with $11 \mathrm{q} 23$ translocation showed markedly poor event free survival than those without 11 q23 translocation [15]. Contrary to these results, the presence of chromosome 11 or 21 abnormalities did not possess a significant prognostic impact on survival in our study, although the latency of AML transformation was significantly shorter than that without chromosome 11 or 21 abnormalities. The prognostic impact of the presence of chromosome 11 or 21 abnormalities, including $11 \mathrm{q} 23$ translocations should be confirmed with a larger study population.

Considering the FAB AML M3 phenotype, our patient cohort included 8 patients who manifested acute promyelocytic leukemia (APL). All of these patients possessed a $P M L / R A R a$ fusion transcript, which was demonstrated by reverse transcriptase PCR. Notably, we found that 4 of the 8 APL patients $(50.0 \%)$ had an additional, single cytogenetic abnormality (inv(6) for 1 patient, del(7) for 1 patient, and +8 for 2 patients). This frequency is higher than that found in previous studies, which report an incidence of $25-41 \%$ [16-20]. Given that the frequency of an additional cytogenetic abnormality in our non-APL patients was 26/40 (65.0\%), which was not significantly different $(P=0.692)$, these results suggest that the good prognosis of an APL manifestation of t-AML might be explained by its unique characteristics, and not due to a low frequency of additional cytogenetic abnormalities. Our APL patients had unique clinical manifestations such as longer latency of t-AML (mean, 59.2 months) and a higher CR rate $(87.5 \%)$ than that reported in previous studies (latency of 2-3 years and CR rates of 60-80\%) [16-19]. These results may be additional evidence for the presence of unique clinical features such as very good prognosis for $\mathrm{t}-\mathrm{AML}$ patients who manifest with APL. Given that the prognosis of $\mathrm{t}-\mathrm{AML}$ patients who manifest with APL proved to be better than the other subtypes in the present study, the prognostic impact of the presence of $t(8 ; 21)$ or $\operatorname{inv}(16)$ at the time of $t$-AML diagnosis (a good prognostic factor in de novo AML) is an interesting question that should be further investigated. However, this issue could not be addressed in the present study, because it included only 1 patient with $t(8 ; 21)$ and no patients with inv(16) at the time of $\mathrm{t}$-AML diagnosis. A larger study population would be required evaluate this issue.

Our study had some limitations. First, several molecular prognostic markers such as mutations in NPM1, CEBPA, $D N M T 3 A, I D H 1$, and $I D H 2$ were not evaluated. Since the prognostic value of these markers has been established in patients with normal karyotype de novo AML, this limitation may have negatively influenced the results of this study. Second, the number of patients included in each group was relatively small, which may have affected the statistical power of our study. A comprehensive study with an adequate number of patients is required to confirm our hypothesis.

In conclusion, patients with a t-AML M3 phenotype were identified as having a better prognosis than other subtypes, suggesting that the AML M3 phenotype possesses unique clinical features. Among the possible prognostic factors, karyotype was the strongest prognostic indicator, predicting a poor prognosis for $\mathrm{t}-\mathrm{AML}$ patients with a complex karyotype.

\section{Authors' Disclosures of Potential Conflicts of Interest}

No potential conflicts of interest relevant to this article were reported.

\section{REFERENCES}

1. Pedersen-Bjergaard J, Andersen MK, Johansson B. Balanced chromosome aberrations in leukemias following chemotherapy with DNA-topoisomerase II inhibitors. J Clin Oncol 1998;16: 1897-8.

2. Cortes J, O'Brien S, Kantarjian H, et al. Abnormalities in the long arm of chromosome 11 (11q) in patients with de novo and secondary acute myelogenous leukemias and myelodysplastic syndromes. Leukemia 1994;8:2174-8.

3. Quesnel B, Kantarjian H, Bjergaard JP, et al. Therapy-related acute myeloid leukemia with $\mathrm{t}(8 ; 21)$, inv(16), and $\mathrm{t}(8 ; 16)$ : a report on 25 cases and review of the literature. J Clin Oncol 1993;11:2370-9.

4. Leone G, Mele L, Pulsoni A, Equitani F, Pagano L. The incidence of secondary leukemias. Haematologica 1999;84:937-45.

5. Pui CH, Relling MV, Rivera GK, et al. Epipodophyllotoxin-related acute myeloid leukemia: a study of 35 cases. Leukemia 1995; 9:1990-6.

6. Michels SD, McKenna RW, Arthur DC, Brunning RD. Therapyrelated acute myeloid leukemia and myelodysplastic syndrome: a clinical and morphologic study of 65 cases. Blood 1985;65: 1364-72.

7. Singh ZN, Huo D, Anastasi J, et al. Therapy-related myelodysplastic syndrome: morphologic subclassification may not be clinically relevant. Am J Clin Pathol 2007;127:197-205.

8. Grossmann V, Schnittger S, Kohlmann A, et al. A novel hierarchical prognostic model of AML solely based on molecular mutations. Blood 2012;120:2963-72.

9. Kuhnl A, Grimwade D. Molecular markers in acute myeloid leukaemia. Int J Hematol 2012;96:153-63.

10. Kayser S, Dohner K, Krauter J, et al. The impact of therapy- related acute myeloid leukemia (AML) on outcome in 2853 adult patients with newly diagnosed AML. Blood 2011;117:2137-45.

11. Antonijevic N, Suvajdzic N, Terzic T, et al. Favourable prognostic factors in therapy related acute myeloid leukaemia. Srp Arh Celok Lek 2011;139:347-52.

12. Schoch C, Kern W, Schnittger S, Hiddemann W, Haferlach T. Karyotype is an independent prognostic parameter in therapyrelated acute myeloid leukemia (t-AML): an analysis of 93 patients 
with t-AML in comparison to 1091 patients with de novo AML. Leukemia 2004;18:120-5.

13. Huh HJ, Lee $\mathrm{SH}$, Yoo $\mathrm{KH}$, et al. Therapy-related myeloid neoplasms in 39 Korean patients: a single institution experience. Ann Lab Med 2013;33:97-104.

14. Smith SM, Le Beau MM, Huo D, et al. Clinical-cytogenetic associations in 306 patients with therapy-related myelodysplasia and myeloid leukemia: the University of Chicago series. Blood 2003;102:43-52.

15. Shim H, Chi HS, Jang S, et al. Therapy-related acute leukemia in breast cancer patients: twelve cases treated with a topoisomerase inhibitor. Korean J Hematol 2010;45:177-82.

16. Andersen MK, Larson RA, Mauritzson N, Schnittger S, Jhanwar SC, Pedersen-Bjergaard J. Balanced chromosome abnormalities $\operatorname{inv}(16)$ and $t(15 ; 17)$ in therapy-related myelodysplastic syndromes and acute leukemia: report from an international workshop. Genes Chromosomes Cancer 2002;33:395-400.

17. Detourmignies L, Castaigne S, Stoppa AM, et al. Therapy-related acute promyelocytic leukemia: a report on 16 cases. J Clin Oncol 1992;10:1430-5.

18. Beaumont M, Sanz M, Carli PM, et al. Therapy-related acute promyelocytic leukemia. J Clin Oncol 2003;21:2123-37.

19. Pulsoni A, Pagano L, Lo Coco F, et al. Clinicobiological features and outcome of acute promyelocytic leukemia occurring as a second tumor: the GIMEMA experience. Blood 2002;100:1972-6.

20. Duffield AS, Aoki J, Levis M, et al. Clinical and pathologic features of secondary acute promyelocytic leukemia. Am J Clin Pathol 2012;137:395-402. 